\title{
Aux frontières du « petit » salariat public et de son encadrement : de nouveaux usages des concours?
}

Close to the small wage public sector and its management: towards new uses of competitive examinations for public employment?

\section{Cédric Hugrée}

\section{OpenEdition \\ Journals}

Édition électronique

URL : http://journals.openedition.org/travailemploi/5340

DOI : 10.4000/travailemploi.5340

ISSN : $1775-416 \mathrm{X}$

Éditeur

DARES - Ministère du Travail

Édition imprimée

Date de publication : 15 septembre 2011

Pagination : 67-82

ISSN : 0224-4365

Référence électronique

Cédric Hugrée, « Aux frontières du « petit » salariat public et de son encadrement : de nouveaux usages des concours? », Travail et Emploi [En ligne], 127 । juillet-septembre 2011, mis en ligne le 09 mai 2012, consulté le 19 avril 2019. URL : http://journals.openedition.org/travailemploi/5340 ; DOI : $10.4000 /$ travailemploi.5340 


\title{
Aux frontières du " petit " salariat public et de son encadrement : de nouveaux usages des concours?
}

\author{
Cédric Hugrée (*)
}

Les emplois de catégorie $B$ de la fonction publique sont aujourd'hui à l'aube de profondes transformations réglementaires et sociales. D'un côté, l'instauration d'un nouvel espace statutaire de la catégorie $B$ risque de transformer progressivement la sociologie de ces fonctionnaires intermédiaires. De l'autre, la condition de diplôme fondant juridiquement la hiérarchie des catégories de fonctionnaires est mise à mal par des candidatures de plus en plus nombreuses des diplômés de l'enseignement supérieur en direction de ces "petits» concours. Comment penser le positionnement social et les carrières de ces fonctionnaires, recrutés pour assurer "des fonctions d'application et de rédaction», avec un baccalauréat? En défendant une lecture "catégorielle» du salariat public contemporain, ce texte revient tout d'abord sur l'érosion sociale qui affecte ces professions à partir des enquêtes FQP 1993 et FQP 2003. Il aborde ensuite, à partir d'une étude de cas ethnographique, la question des candidatures des diplômés de l'enseignement supérieur long à ces concours. Ces candidatures n'apparaissent pas seulement comme la conséquence d'une abstraite "inflation scolaire». Elles s'inscrivent au contraire au croisement d'une socialisation universitaire inachevée et de carrières concourantes le plus souvent contrariées.

Dans un contexte marqué par une transformation institutionnelle importante de l'État et des manières de gouverner (BÉZĖs, 2009), par la modification des conditions de recrutement des fonctionnaires ${ }^{(1)}$ et par la baisse des recrutements dans la Fonction Publique d'État (FPÉ), certains lieux s'imposent comme décisifs pour l'investigation sociologique de l'emploi public. Et, face aux instigateurs des réformes actuelles de l'administration, au «laboratoire de la modernisation de l'emploi public»(2) qu'incarne la Fonction Publique Territoriale (FPT) et aux principales victimes de la réduction de l'emploi public que sont les concours de catégorie A (3), la sociologie des fonctionnaires de catégorie B semble n'avoir que peu d'atouts heuristiques...

Catégorie administrative intermédiaire entre les agents de catégorie $\mathrm{C}$ et ceux de catégorie $\mathrm{A}$ dans le statut général de la fonction publique de 1983 et 1984, celle-ci se révèle protéiforme à l'échelle historique.

(*) cedric.hugree@esu.cresppa.cnrs.fr

(1) On fait ici référence au Réexamen général des contenus des concours (RGCC) qui s'appuie sur certaines recommandations des rapports Desforges (2008) et dont le plan d'action est définie par la circulaire du 20 juin 2008, Ministère du budget, des comptes publics et de la fonction publique, DGAFP.

(2) Selon les mots du Premier Ministre et du Ministre de la fonction publique, cité par Émilie BILAND (2010b).

(3) Ces derniers représentent $70 \%$ des candidats effectivement recrutés par concours externe en 2007; les enseignants représentant $85 \%$ des candidats effectivement recrutés en catégorie A (BOUNAKHLA, 2008, p. 434).
L'enquête historique de Jean Le Bihan révèle ainsi un véritable «groupe professionnel» émergent au $\mathrm{XX}^{\mathrm{e}}$ siècle (LE BIHAN, 2008, p. 57) à la position hiérarchique située entre le haut et le bas de l'organisation administrative, c'est-à-dire distinct des employés et subordonnés à leurs supérieurs (hauts fonctionnaires). Ils sont d'ailleurs marqués pour l'historien par une ambition commune mais impossible de promotion au $\mathrm{XIX}^{\mathrm{e}}$ siècle : «[...] les fonctionnaires intermédiaires à mesure qu'ils se rapprochent de leurs supérieurs sur le plan technique et administratif, se heurtent de plus en plus à eux sur le plan social et statutaire.» (Ibid. pp. 223-260) «Salaires et indemnités ne sont sans doute pas mirifiques, mais ils suffisent à distinguer dans les faits et les esprits, les fonctionnaires intermédiaires de leurs employés qui seuls ont connu la gêne» (Ibid. pp. 330-331). La socio-histoire des receveurs des Postes sous la Quatrième République réalisée par Odile Join-Lambert montre cependant, qu'à l'échelle de l'administration postale, le portrait de receveur moyen est impossible à dresser tant «sa position sociale change selon la taille de la localité et sa sociologie» (JoIn-LAmBerT, 2005, p. 14). Ces fonctionnaires occupent également pour l'auteur une "position intermédiaire entre les agents d'exécution, les usagers et les responsables des directions départementales» (Ibid.) et semblent bien marqués par une communauté d'aspirations à la promotion interne. Mais elle note que ce sont d'abord et surtout les organisations syndicales et professionnelles qui ont favorisé l'unification progressive d'un corps 
faisant se rencontrer sur une même grille d'avancement et sous un même statut des employés de petits bureaux postaux et des cadres de l'administration postale. L'unité et la mobilité des receveurs des Postes trouvent d'ailleurs ensemble leurs limites dans l'étude des déterminants des carrières. Leurs origines sociales, scolaires et géographiques sont au principe d'une différenciation importante des itinéraires professionnels qui nuancent l'idée de "possibilités d'ascension sociale permises par la promotion interne de la Libération à la fin des années $1950 »$; «le verrou d'accès à la catégorie des cadres [s'étant] effectué de manière significative dans un intervalle de dix ans» (ibid. pp. 31-32).

Qu'en est-il aujourd'hui? Comment penser le positionnement social et les carrières des fonctionnaires de catégorie $\mathrm{B}$, concours initialement conçus pour recruter formellement les agents qui assurent «des fonctions d'application et de rédaction», pour l'essentiel au niveau du baccalauréat?

L'actualisation d'une lecture «catégorielle» du salariat public contemporain semble d'autant plus nécessaire que ces concours et ces emplois sont aujourd'hui à l'aube de profondes transformations réglementaires et sociales. D'un côté, l'instauration d'un Nouvel espace statutaire (NES) de la catégorie $\mathrm{B}^{(4)}$ transforme progressivement la sociologie de ces fonctionnaires intermédiaires en proposant le basculement d'anciens corps de catégorie B en catégorie A (infirmière's des trois fonctions publiques notamment), la fin de la distinction entre les corps «B-Type administratif» recrutant au niveau du baccalauréat et les corps «B-Classement Indiciaire Intermédiaire $(\mathrm{CII}) »($ dits $« \mathrm{~B}+»)$ créées suite aux accords «Durafour» (5), ainsi que des élévations sensibles des salaires en début et fin de carrière.

De l'autre, la condition de diplôme fondant juridiquement la hiérarchie des catégories de fonctionnaires est mise à mal par des candidatures de plus en plus nombreuses de diplômés de l'enseignement supérieur en direction de ces «petits» concours. Les concours de catégorie B de la FPÉ voient en effet aujourd'hui leurs lauréats souvent issus de l'enseignement supérieur long. À la session 2007, pour 5054 postes de catégorie B ouverts au concours externe, on dénombrait 93858 candidats présents aux épreuves

(4) Cette réforme s'inscrit dans le $3^{\mathrm{e}}$ volet de conclusion du 21 février 2008 en vue de la revalorisation de la grille indiciaire de la catégorie B. Cet accord a été signé par la CFDT, la CFTC, la CFE-CGC, et l'UNSA; la CGT et la FSU ayant refusé de signer cet accord. Selon le ministère du budget, des comptes publics et de la fonction publique, cette réforme vise à « regrouper en une grille unique harmonisée et revalorisée les personnels de catégorie B qui relèvent actuellement de grilles indiciaires différentes et de fluidifier leur carrière.»

(5) Protocole d'accords conclu le 9 février 1990 sur la rénovation de la grille des classifications et des rémunérations des trois fonctions publiques (fonction publique de l'État, fonction publique hospitalière et fonction publique territoriale), accords dits «Accords Durafour». et finalement 6344 candidats recrutés. Parmi eux, près d'un sur deux (49\%) avait un diplôme égal ou supérieur à bac +2 et plus d'un sur trois $(35 \%)$ avait au moins un bac +3 (BOUNAKHLA, 2009).

\section{Encadré
La combinaison de l'analyse secondaire des enquêtes FQP(1) et le suivi ethnographique d'une enquêtée

Ce texte s'appuie sur l'exploitation secondaire des enquêtes FQP 1993 et FQP 2003 (Goux, 2010; Monso, THÉVENOT, 2010). Les traitements réalisés reposent sur une définition du salariat public notamment permise par la variable «Statut des salariés (CHPUB)» des deux enquêtes, soit 3017 salariés actifs pour l'enquête FQP 1993 et 7006 salariés actifs pour celle de 2003. Ces exploitations secondaires sont ici complétées par l'analyse d'entretiens répétés avec une enquêtée, entretiens réalisés entre 2005 et 2010, au moment de sa transition professionnelle à l'issue de ses études à l'université de Nantes.

La confrontation d'une sociographie transversale des rapports entre les différentes composantes du salariat public (construite depuis les catégories statistiques) à un cheminement individuel (construit depuis les catégories d'une ethnographie longitudinale) donne l'occasion de «penser par cas» (PAsseron, Revel, 2005) les nouvelles conditions d'accès aux concours; c'est-à-dire de tenter d'élucider ensemble la singularité d'une candidature et d'un parcours et la configuration sociale qui les rendent possible. La combinaison de l'exploitation secondaire de données quantitatives et de l'entretien ethnographique (BEAUD, 1996) rend alors simultanément visible les évolutions structurelles qui façonnent les Professions intermédiaires (PI) administratives de la FP ainsi que les pratiques scolaires, les représentations sociales et les aspirations personnelles qui, à l'échelle biographique et individuelle, déterminent concrètement l'accès à un des statuts qui composent cette catégorie sociale sans que ces éléments décisifs des nouvelles candidatures aux emplois publics ne soient repérables dans les fichiers des enquêtes de la statistique publique. Le croisement des sources et le recours à différents types de traitements sont aussi l'occasion de nuancer et d'affiner les catégories statistiques qui ordonnent et délimitent strictement l'espace social observé. Enfin, en opérant un retour statistique sur les PI administratives de la FP en 1993 et en 2003, on focalise l'attention sur deux dates précises qui permettent d'approcher les effets de la seconde explosion scolaire sur un ensemble de professions du secteur public. Le récit du parcours de Camille, ayant quitté l'université en 2002 et recrutée en 2006, confirme la centralité de l'expansion universitaire des années 1990-2000 pour la sociologie des générations des salariés de la fonction publique. Mais la présentation de ce cas d'enquête invite, en même temps, à mieux apprécier à l'échelle d'une administration et d'un corps, les calendriers d'arrivée des diplômés de l'enseignement supérieur long au sein des différentes catégories B de la FP; les décalages chronologiques pouvant alors être vus comme des révélateurs de la transformation en actes de la hiérarchie des concours.

(1) Formation Qualification professionnelle. 
Au-delà de ces constats généraux, les éléments manquent cependant pour caractériser plus précisément cette composante du salariat public contemporain. Ainsi, l'étude des parcours d'entrée dans la fonction publique faite à partir des enquêtes Génération 98 (Céreq) et Formation Continue 2000 (Insee), indique-t-elle de nettes différences dues au sexe, à la formation initiale ou encore à la situation familiale dans la construction des itinéraires d'accès à l'emploi public (Di Paola, Moullet, 2003). Mais, rien dans ces résultats, pourtant inédits, ne permet de distinguer les effets des variables traditionnelles de la sociologie (sexe, diplôme, origine sociale, etc.) dans l'accès aux catégories d'emplois statutaires (A, B, C).

La mobilité catégorielle des agents titulaires de l'État est certes un sujet classique des analyses quantitatives des mobilités des fonctionnaires. Dans ces études, le recours à la nomenclature des PCS s'impose pour approcher leurs mobilités professionnelles (PAURon, 2003, pp. 101-102). Ces analyses quantitatives se heurtent alors malgré tout à l'histoire salariale des reclassements de certains corps (instituteurs devenant professeurs des écoles, etc.) qui rendent complexe la quantification des passages de catégorie à une autre et son interprétation.

Faut-il pour autant abandonner l'ambition d'une sociologie quantitative de cette partie du salariat public depuis les sources de la statistique publique? Il semble au contraire que l'intérêt de rapporter cette catégorie particulière de fonctionnaires (catégorie B) à sa catégorie sociale au sein de la nomenclature PCS (Professions intermédiaires administratives de la fonction publique) soit aussi de penser ces agents de la fonction publique dans le cadre d'une sociologie des groupes et des rapports sociaux.

Les données publiées par Thomas Amossé (2011, p. 39) présentent d'abord les Professions intermédiaires administratives de la fonction publique (PI administratives de la FP) comme un groupe frontière avec les cadres supérieurs en 1982 comme en 2002. Mais, fait notable, ces emplois se situent désormais légèrement du côté «non-cadre» de la frontière qui sépare les cadres de l'ensemble du salariat français là où ils figuraient, vingt ans plus tôt, du côté «cadre» de cette frontière. Les résultats de Jérôme Deauvieau et Céline Dumoulin (2009), centrés sur les rapports entre les différentes composantes des professions intermédiaires en 1985 et en 2003, révèlent alors un groupe «en déclin» à l'intérieur du «pôle administratif et commercial» des professions intermédiaires françaises. Certains des traits sociologiques saillants des fonctionnaires intermédiaires contemporains invitent cependant à formuler l'hypothèse que l'objectivation de leur position sociale passe autant par le repérage de leurs convergences et de leurs divergences avec les autres salariés du public que par une comparaison avec les différentes composantes des professions intermédiaires. Ce texte propose donc d'étudier dans un premier temps l'évolution relative du positionnement social des PI administratives de la FP à l'intérieur du salariat public à partir des enquêtes Formation qualification professionnelle 1993 et 2003 (FQP) ( $c f$. encadré). En faisant des PI administratives de la FP le cœur de notre propos, on souhaite d'abord appréhender la distance sociale qui sépare désormais ces fonctionnaires intermédiaires du «petit» salariat public. C'est aussi une première comparaison des «gens du public » (Singly, THÉLot, 1988) en fonction de leurs trajectoires scolaires et sociales que l'on donne à voir. Cette photographie statistique est complétée, dans un second temps, par le récit de la trajectoire scolaire et sociale d'une jeune licenciée d'Histoire ayant accédé à ce type d'emploi à l'Agence nationale pour l'emploi (ANPE) en 2006, plusieurs années après sa sortie de l'université.

\section{Polarisation du salariat public et prolétarisation des professions intermédiaires administratives de la fonction publique?}

À la lecture des récents travaux sur les professions intermédiaires (Deauvieau, Dumoulin, 2009, 2010), la catégorie socioprofessionnelle des PI administratives de la FP apparait tout d'abord comme un groupe globalement stable numériquement dans la structure sociale française, passant de $9 \%$ de l'ensemble des professions intermédiaires en 1982 à $8 \%$ en 2002 (ce qui représente près de 60000 individus supplémentaires). Constituant, avec les professions intermédiaires administratives et commerciales des entreprises, le «pôle administratif et commercial des professions intermédiaires », les PI administratives du public s'en distinguent cependant par des origines sociales plus populaires, par leur vieillissement, ainsi que par leur pente démographique puisque les PI administratives et commerciales du privé ont, elles, crû de 5 points sur la même période. Les auteurs soulignent également que les carrières de ces fonctionnaires intermédiaires divergent de celles de leurs homologues du privé, non pas tant dans leurs chances de connaître une mobilité ascendante (respectivement $14 \%$ et $13 \%$ ) que dans celles de faire face à une mobilité descendante $(13 \%$ pour les PI administratives et commerciales du privé contre $7 \%$ pour les PI du public). Ils concluent ainsi que ces carrières ascendantes «s'expliquent par la permanence de voies de promotion professionnelle vers les catégories A de la fonction publique» (Deauvieau, Dumoulin, 2010, p. 67), dont on sait qu'elle touche essentiellement les filières administratives et non enseignantes de ce secteur (Gollac, 2005, p. 54). 


\section{Des fonctionnaires de l'État âgés et toujours en promotion professionnelle}

À l'échelle du salariat public, la part des PI administratives du public âgés de plus de 35 ans a augmenté de 7 points entre 1993 et 2003; aujourd'hui seulement un membre des PI administratives du public sur cinq (21\%) a moins de 35 ans. Cette proportion a augmenté de 7 points entre 1993 et 2003 pour les professeurs et professions scientifiques $(31 \%)$, de 3 points pour les instituteurs et assimilés $(41 \%)$ et pour les policiers et militaires (58 \%). Si les PI administratives de la FP ne sont pas les seules catégories de salariés du public à connaître un vieillissement relatif, la proportion de moins de 35 ans et son évolution les placent dans une dynamique proche de celle des cadres de la FP qui ne comptent plus que $22 \%$ de moins de 35 ans (baisse de 4 points).

Ces éléments peuvent se lire à la lumière du nombre de salariés du public qui ont accédé à leur poste par promotion interne au moment de l'enquête. Bien qu'en recul pour les cadres et les PI de la FP entre 1993 et 2003 ( -13 et - 11 points), la part de ces salariés ayant accédé à leur poste par promotion demeurent importantes ( $25 \%$ et $28 \%$ ), ce qui en fait deux des catégories de salariés du public les plus «promues» (17\% en moyenne).

L'appartenance des PI administratives de la FP à l'administration nationale (et non territoriale ou hospitalière) est enfin un trait qui les fait nettement se distinguer des autres salariés du public. En 1993, $71 \%$ des PI du public sont salariés de l'administration nationale, ce qui les place parmi les trois catégories où figuraient le plus de salariés de l'État avec les cadres de la FP $(73 \%)$ et les policiers et militaires $(75 \%)$. Dix ans plus tard, les salariés de l'État sont encore majoritaires parmi les PI administratives du Public mais leur proportion a baissé de façon importante ( -15 points), dans une proportion identique à celle des cadres de la FP ( -16 points). La catégorie des policiers et militaires voit quant à elle sa proportion de salariés de l'État se maintenir ( -2 points). On ne s'étonnera pas dans ces conditions de voir les PI administratives de la FP figurer en 1993 et 2003 dans le haut de la moyenne des catégories des salariés du public étant fonctionnaires et non contractuels : près de $90 \%$ d'entre eux sont titulaires ou stagiaires. Mais, on le voit également, le fort développement de la fonction publique territoriale sur la période étudiée (RAYNAUD, 2003) contribue aussi à redessiner la carte administrative et tutélaire du groupe de fonctionnaires étudié.

\section{Un groupe mixte et de plus en plus diplômé... mais qui peine à se rapprocher du haut du salariat public}

Cette première cartographie positionnant les PI administratives de la FP au regard des autres salariés du secteur public doit être complétée par l'étude spécifique de la répartition sexuée et de la composition scolaire. Si l'on sait la féminisation de l'emploi public importante, celle-ci n'en reste pas moins très nettement polarisée selon des logiques proches des emplois de service (Rouban, 2009; Chenu, 2005 [1994]). Regardé conjointement avec la part des titulaires de bac +2 et plus, l'espace du salariat public apparait bien divisé selon ces deux logiques, en 1993 (graphique 1, figure de gauche) et peut-être plus encore en 2003 (graphique 1, figure de droite). À droite de ces graphiques, les catégories socioprofessionnelles de l'emploi public appartenant au groupe socioprofessionnel des cadres et professions intellectuelles supérieures, et quelques professions intermédiaires, où le bac +2 et plus s'est progressivement établi comme la norme scolaire et/ou réglementaire de recrutement; à gauche, les emplois d'ouvriers et d'employés où la présence de tels profils scolaires reste exceptionnelle, en 1993 comme en 2003. Mais la distribution sexuée de différentes catégories de salariés de la fonction publique structure elle-même largement les différents pôles opposant d'un côté, les métiers de la santé et du social, de l'enseignement primaire, les agents de service très majoritairement féminins, et de l'autre les emplois de policiers et militaires et d'ouvriers, massivement masculins.

Si l'on s'attarde désormais sur les PI administratives de la FP, on constate qu'entre 1993 et 2003, ils accompagnent, dans le même mouvement vers la droite, les autres catégories de salariés de la FP appartenant aux professions intermédiaires ${ }^{(6)}$. Cependant, bien qu'ils semblent s'éloigner dans leur composition scolaire des employés et agents de service de la FP, ils demeurent encore distants du groupe de salariés se rapprochant désormais des professeurs et professions scientifiques.

Ainsi, l'évolution de la composition sexuée et scolaire du salariat public entre 1993 et 2003 laisse entrevoir une véritable tripartition : à droite, un ensemble de professions diplômées du secteur public où la proportion de femmes se maintient voire augmente à l'intérieur de catégories hier très masculines, à l'image des cadres de la FP. À gauche, on observe les salariés les moins diplômés et plus nettement séparés par des logiques de genre avec, en haut, le pôle des employés dont les PI administratives de la FP se détachent par le biais de la proportion de bac +2 et plus et, en bas, celui des ouvriers et policiers. À fois vieillissantes et issues de la promotion professionnelle interne au secteur public, les PI administratives de la FP semblent avoir été affectées par une élévation modérée du

(6) Il s'agit ici des Professions de la santé et du travail social, des professeurs des écoles (et assimilés). Les autres PI du Public sont constituées essentiellement de techniciens, d'agents de maîtrise, de contremaîtres auxquels s'ajoutent quelques enquêtés issus du clergé. 
Graphique 1 : Les professions intermédiaires administratives de la fonction publique dans l'espace du salariat public en 1993 et en 2003 selon le niveau de diplôme et le sexe

1993

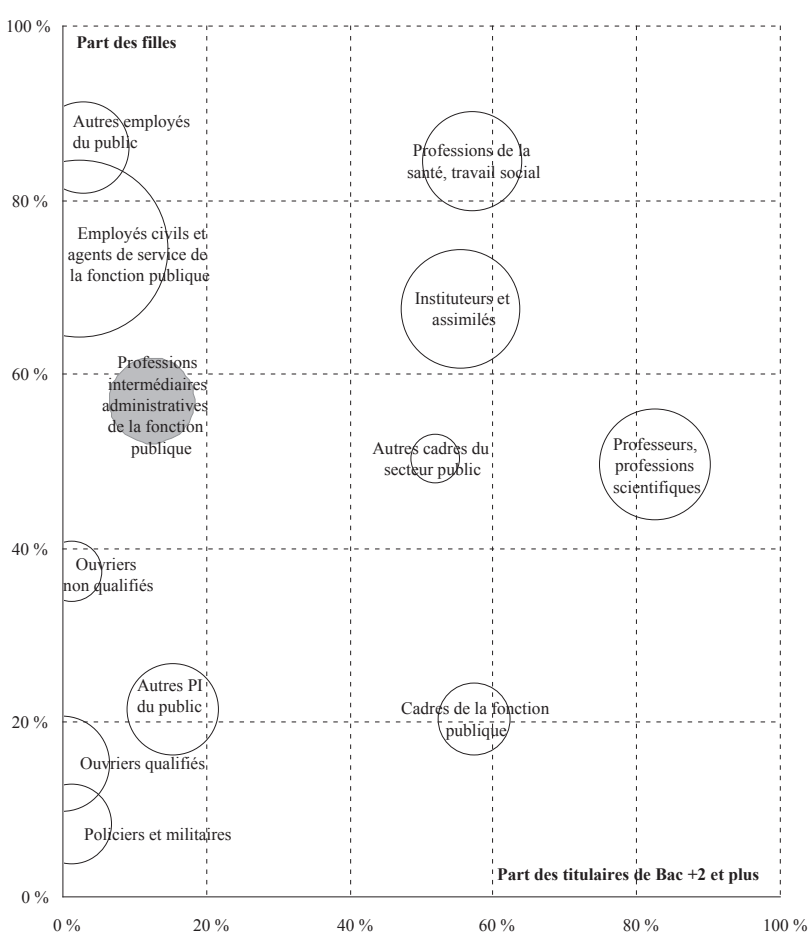

2003

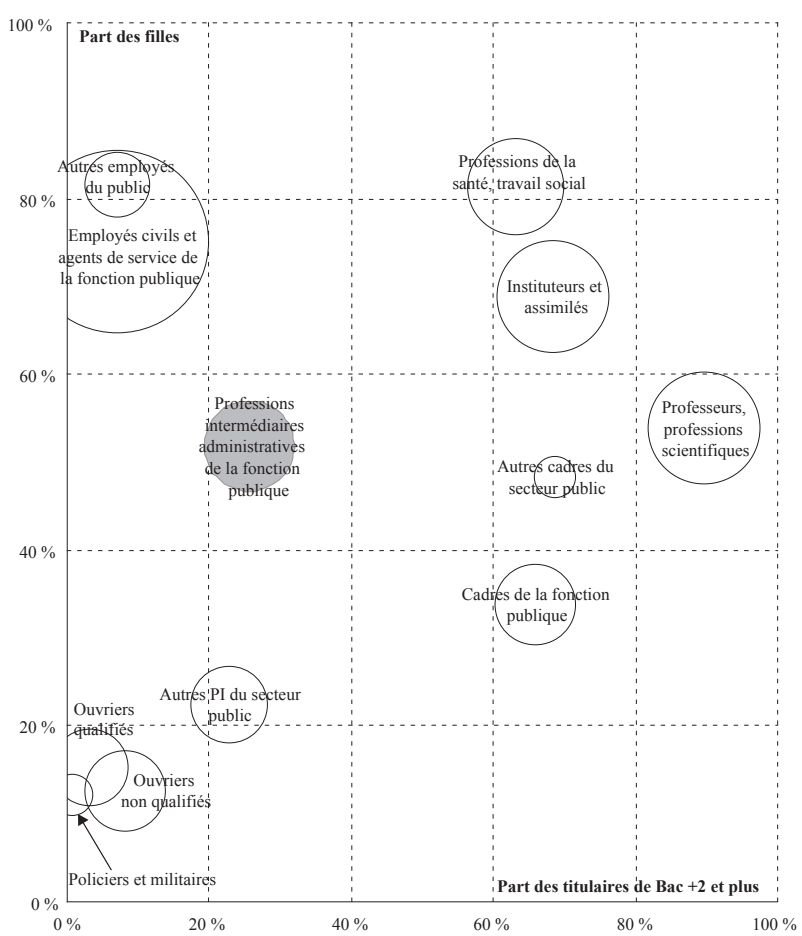

Lecture : Pour 100 membres des PI administratives de la FP en 2003 (figure de droite), 26 ont au moins un bac +2 (abscisses) et 52 sont des filles (ordonnées). En 1993, ils étaient $12 \%$ à avoir au moins un bac +2 et $57 \%$ étaient des filles. Ces deux taux sont calculés pour l'ensemble des salariés du secteur public, quelle que soit leur origine sociale. En revanche, la surface de chaque cercle est proportionnelle à la part de chacune des CS dans le salariat public.

Champ : Actifs appartenant au secteur public en 1993, en 2003.

Sources : Enquêtes FQP 1993 et FQP 2003, Insee (Diffusion : Centre Maurice Halbwachs).

niveau de diplôme de leurs membres. Et si elles ne se présentent plus comme le centre de gravité des professions intermédiaires (DeAuvieau, Dumoulin, 2009), ces professions semblent bien occuper, à l'échelle du salariat public, une position frontière entre un «petit» salariat public relativement stable et un encadrement où la recomposition scolaire est plus nette.

\section{Polarisation du salariat public et stabilisa- tion des PI administratifs du public}

Pour compléter ces premiers éléments, on a procédé à une double analyse de correspondance multiple, ici superposée sur un plan (graphique 2). Cette première méthode d'analyse multivariée permet tout d'abord d'appréhender graphiquement l'espace du salariat public français et les structures d'opposition entre les différents pôles qui le composent. Réalisée sur les données des deux enquêtes FQP 1993 et 2003, l'analyse permet de mieux comprendre l'évolution de la structure des écarts à l'intérieur de l'ensemble étudié, et par analogie, d'appréhender les frontières sociales entre les différents groupes constitutifs des salariés du secteur public, en 1993 et en 2003. Le plan qui résulte de l'analyse porte sur les principales oppositions qui organisent le salariat public à ces deux dates. La structure d'opposition des quatre pôles qui émergent de ces deux analyses n'évolue pas de façon fondamentale, même si des modifications sont à relever pour certaines variables.

La catégorie socioprofessionnelle ne prend pas part active à l'analyse et, en 2003, ce sont d'abord les variables qui résultent principalement des différentes structures de l'emploi public qui organisent le premier axe : d'un côté, à l'est, on trouve le fait d'être en CDD ou en vacation ( $12 \%$ de contribution à l'axe), d'être en premier poste ( $8 \%$ ), d'avoir moins de trente-cinq ans ( $8 \%$ ), d'être une femme $(7 \%)$; de l'autre, à l'ouest, on trouve le fait d'être un homme $(9 \%)$, d'avoir accédé à son poste par promotion interne $(6 \%)$, de travailler dans une administration nationale $(5 \%)$. Le deuxième axe rend davantage visible les hiérarchies sociales et professionnelles à l'intérieur du secteur public. Au nord, on trouve le fait d'avoir un conjoint ouvrier $(14 \%)$, de ne pas posséder de diplôme ou un CEP $(13 \%)$, un père ouvrier ( $9 \%)$, d'être titulaire d'un CAP ou BEP $(5 \%)$. Au sud, on voit apparaître la possession d'un bac +2 ou plus $(15 \%)$, le fait d'avoir un conjoint cadre $(9 \%)$ et d'avoir un père cadre supérieur $(8 \%)$ ou profession intermédiaire $(5 \%)$. 
Graphique 2 : La structure sociale du salariat public en 1993 et en 2003

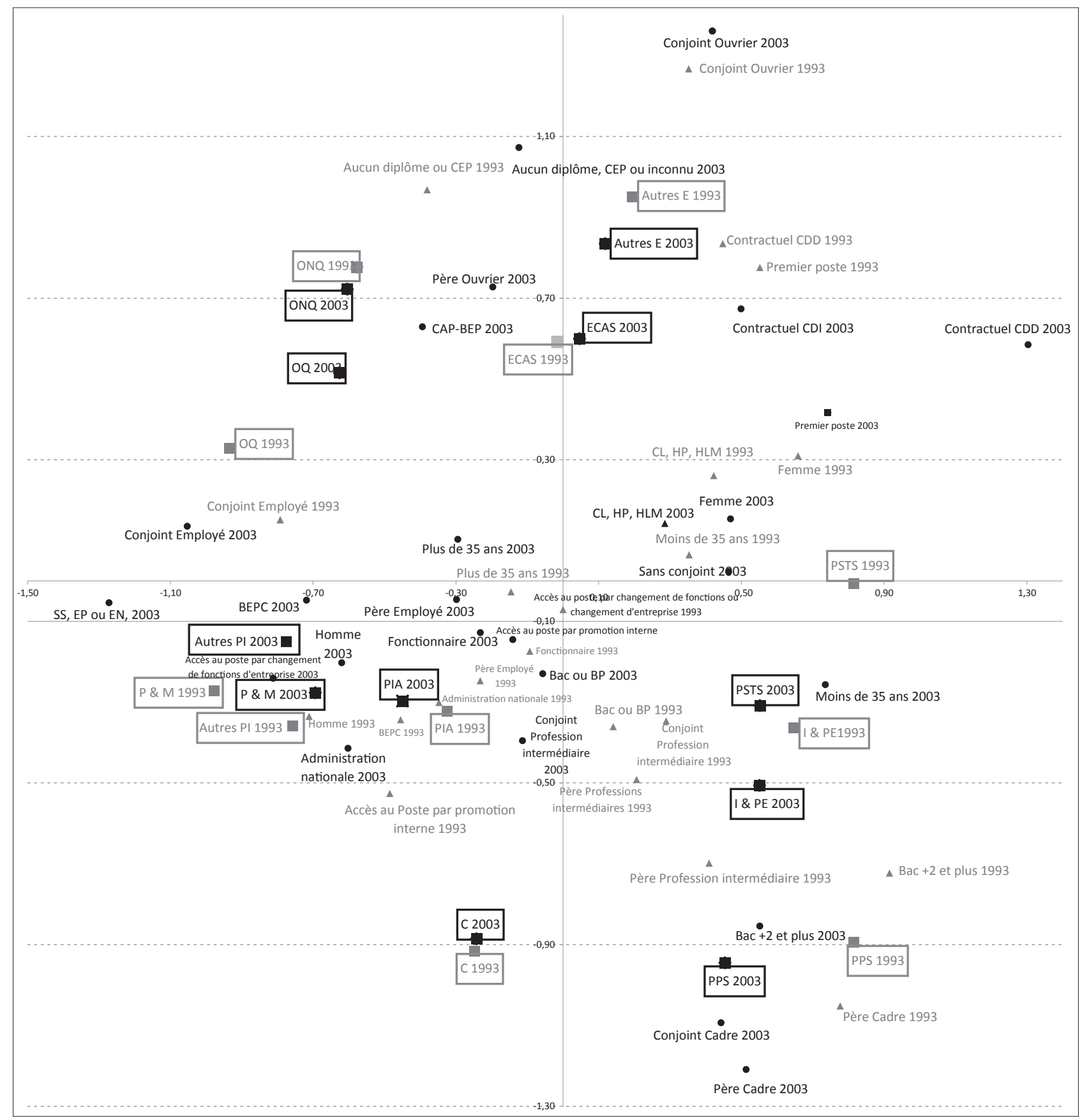

Note : Ce graphique est construit à partir de deux analyses factorielles de correspondance multiples dont les plans ont été superposés de façon à rendre compte des structures du salariat public entre 1993 et 2003. Cette représentation indique l'évolution des structures du salariat public aux dates et non une mesure des évolutions au sein d'un même plan.

Légende : ONQ : ouvriers non qualifiés; OQ : Ouvriers qualifiés; E : employés; ECAS : employés civils et agents de service; P \& M : Policiers et militaires; PIA : Professions intermédiaires administratives; I \& PE : Instituteurs, Professeurs des écoles et assimilés; PSTS : Professions de la santé et du travail social; $\mathrm{C}$ : Cadres; PPS : Professeurs et professions scientifiques.

L'axe 1 (abscisses) porte $27 \%$ d'inertie et l'axe 2 (ordonnées) $22 \%$ pour l'enquête FQP 1993 (modalités en gris). L'inertie est respectivement de $26 \%$ et $24 \%$ pour les axes des abscisses et des ordonnées de l'enquête FQP 2003 (modalités en noir).

Lecture : Les catégories socio-professionnelles encadrées correspondent à des projections sur le plan en tant que variables supplémentaires. Les libellés (gris ou noirs) correspondent aux modalités actives des variables retenues. On tente ainsi de caractériser les rapports entre les différents groupes composant le salariat public entre 1993 et 2003. Certains libellés n'ont pas été reproduits pour davantage de lisibilité.

Champ : Actifs appartenant au secteur public en 1993 et en 2003.

Sources : enquêtes $F Q P 1993$ et $F Q P$ 2003, Insee (Diffusion : Centre Maurice Halbwachs).

Sur cette carte, les sous-groupes sociaux et professionnels qui composent le salariat public apparaissent, en 1993 comme en 2003, séparés en pôles. Sans qu'elle indique une quelconque intensité des écarts entre ces pôles, l'évolution des différents éléments qui structurent ces oppositions est l'indice de certaines recompositions sociales dans le salariat public.

On retiendra ici que les variables liées aux formes d'emplois (statutaires ou contractuelles) contribuent nettement moins aux oppositions des deux 
axes en 1993 qu'en 2003 alors que celles liées au sexe et au niveau d'étude «bac +2 et plus » contribuent davantage en 1993 qu'en 2003, ce qui invite à préciser davantage les diplômes se situant au-delà de ce seuil.

$\mathrm{Si}$, entre ces deux dates, les PI administratives de la FP occupent une position globalement stable au centre ouest du graphique, c'est loin d'être le cas des autres catégories socioprofessionnelles qui appartiennent aux professions intermédiaires, à l'image des professions de la santé et du travail social, et des instituteurs (et assimilés), pour lesquels le rapprochement avec les professions qualifiées situées en haut du salariat public est souligné dans plusieurs travaux récents (Amossé, 2011, p. 40).

Ce que laisse finalement apparaître cette première cartographie du salariat public en 1993 et en 2003, c'est une progressive polarisation en sous-groupes faisant aujourd'hui se rapprocher d'une part les ouvriers des employés de la FP, d'autre part les professions de la santé et du travail social des instituteurs (et assimilés) et mêmes des professeurs et professions scientifiques, et enfin les PI administratives de la FP des policiers et militaires. De ce mouvement émergent plus nettement, au nord, les emplois les moins qualifiés du secteur public, au sud-ouest, les emplois administratifs ou ceux liés aux fonctions régaliennes de l'État et au sud-est les emplois les plus qualifiés, qui rappellent ceux que Pierre Bourdieu avait désignés comme incarnant la «main gauche de l'État». Ce mouvement semble alors accompagné d'une évolution des critères de structuration du salariat public qui passent du sexe et du diplôme ${ }^{(7)}$ au statut.

Dans cette configuration, les PI administratives de la FP continuent d'occuper une position singulière à l'intérieur de l'emploi public : à la fois entre les policiers et militaires et les instituteurs en 1993 , et davantage désormais entre les professions de la santé et du social sur l'axe 1, les employés civils et agents de service et les cadres de la fonction publique sur l'axe 2. Proches, à l'échelle du salariat public, des policiers et militaires, il semble donc qu'on puisse (avec les critères de 2003) moins les confondre avec les instituteurs (et assimilés) et les professions de la santé et du travail social que cela n'était le cas avec les critères de 1993. De ce même point de vue, elles sont encore plus éloignées des employés civils et agents de service de la FP.

Si l'on souhaite désormais saisir les différentes caractéristiques qui, «toutes choses égales par ailleurs », tendent à faire se distinguer les membres des PI administratives de la FP de l'ensemble des salariés du secteur public, en 1993 et en 2003, on voit que la permanence de leur position sociale

(7) Du moins tel qu'il est défini dans la présente recherche (Bac +2 et plus). entre ces deux dates s'est pourtant accompagnée de changements ( $c f$. tableau 1$)$. Les deux premières analyses de régression confirment certaines spécificités (selon les variables retenues) des PI administratives au regard de l'ensemble du salariat public puisque les pourcentages des paires concordantes sont élevés $(89,3 \%$ pour 1993 et $83,9 \%$ pour 2003$)$. La baisse de ce premier indicateur entre 1993 et 2003 indique malgré tout que les critères premièrement mobilisés pour expliquer le fait d'être PI administratives de la FP en 1993 méritent d'être complétés en 2003.

Les caractéristiques les plus fortement associées aux PI administratives de la FP sont désormais le fait de travailler pour une administration nationale (l'odds ratio ${ }^{(8)}$ des modèles testés passe de 4,0 à 9,8 entre 1993 et 2003), d'avoir un emploi appartenant au groupe des professions intermédiaires cinq années auparavant (l'odds ratio passe de 3,4 à 4,2 entre 1993 et 2003), et d'avoir un ou plusieurs salariés sous ses ordres (l'odds ratio passe de 2,2 à 2,3 entre 1993 et 2003). Toutes choses égales par ailleurs, il est également de moins en moins improbable d'être PI administrative de la FP lorsqu'on accède à son poste par changement de fonction ou de poste (au sein de la même administration ou entreprise) alors qu'il est de plus en plus improbable de l'être par voie de promotion interne.

L'évolution des caractéristiques scolaires des PI administratives de la FP au regard de celle des autres catégories du salariat public tend finalement à révéler différentes générations en partie produites par l'évolution des dispositions réglementaires de recrutement et l'élévation progressive, pour les cohortes les plus récentes, du niveau des candidats aux concours concernés. Ainsi, en 1993, la possession d'un BEPC seul était, toutes choses égales par ailleurs, plus associée aux PI administratives de la FP qu'aux autres salariés du public (l'odds ratio est de 2,2) que celle d'un diplôme supérieur ou égal à bac +2 . Ce dernier niveau de diplôme diminuait, à l'époque, la probabilité d'appartenir à ce groupe au sein du salariat public. La situation semble différente dix ans plus tard même si certains diplômes du bas de la hiérarchie scolaire (tels que le BEPC) demeurent des marqueurs significatifs des PI administratives de la FP au regard des autres salariés du

(8) L'odds ratio (rapport de chances) exprime ici la distance logistique entre les variables qui construisent le modèle «être PI administratives de la FP plutôt que ne pas l'être» (soit ici appartenir aux autres catégories de salariés du secteur public) en 1993 et en 2003 (Tableau 1). Un odds ratio égal à 1 signifierait qu'il n'y a pas de différence entre, par exemple, les proportions de réponses «travaille pour une administration nationale» chez les PI administratives de la FP et chez les autres salariés du public. Un odds ratio supérieur à 1 indiquerait que la proportion de réponses «travaille pour une administration nationale» observée chez les autres salariés du public est moindre que celle observée chez les PI administratives de la FP et inversement pour un odds ratio inférieur à 1 . 
secteur public. En effet, le niveau bac +2 et plus qui influençait négativement la probabilité d'appartenir aux PI administratives de la FP en 1993 devient, toutes choses égales par ailleurs, en 2003, un facteur qui lui est désormais plus fortement associé (l'odds ratio passe de 0,4 en 1993 à 1,5 en 2003). C'est donc bien au cours de la seconde explosion scolaire que s'est amorcé le changement du recrutement scolaire et universitaire d'une partie des fonctionnaires intermédiaires contemporains.

Le dernier modèle testé confirme ces éléments. Mais, en prenant en compte la question de la «mobilité sociale subjective» notamment, il donne à voir un élément supplémentaire de caractérisation de cette composante du salariat public. On observe ainsi qu'en 2003, le fait de déclarer que le niveau ou le statut de l'emploi actuel occupé est plus élevé que celui de son père va de pair, toutes choses égales par ailleurs, avec une probabilité plus forte (odds ratio de 1,37) d'être PI administrative de la FP plutôt qu'une autre catégorie socioprofessionnelle du salariat public. Ce sentiment d'une mobilité sociale intergénérationnelle ascendante plus marqué chez les PI administratives de la FP que chez les autres membres de la FP peut être rapproché d'un autre trait subjectif, qui les distingue également nettement des autres catégories socioprofessionnelles. Tristan Poullaouec et Agnès Pelage (2009) ont en effet remarqué, à partir de l'enquête Histoire de vie-Construction des identités (Insee 2003), que les PI administratives de la FP figurent parmi les dix catégories socioprofessionnelles (CS) déclarant le plus un sentiment de classe $(55 \%)$ mais surtout que les membres de cette CS utilisent nettement plus que les autres l'expression de «classe moyenne» pour exprimer leur appartenance de classe (58\% contre $36 \%$ en moyenne).

Les forts sentiments de mobilité sociale ascendante et d'appartenance aux classes moyennes constituent donc deux traits importants de l'identité sociale des PI administratives de la FP qui contrastent avec leur perte de prestige entrevue précédemment. On peut alors regarder ces traits subjectifs saillants à la lumière des trajectoires sociales, scolaires et professionnelles de leurs membres. Sans revenir sur le débat afférant aux déterminants de la mobilité sociale subjective et aux manières de confronter mobilité objective et mobilité subjective (DuruBellat, KiefFer, 2006; Merllié, 2006), on doit tout d'abord noter que le fait d'avoir un père appartenant à la catégorie d'employé au moment de l'arrêt des études est un facteur qui multiplie, en 2003, par 1,33 les chances d'appartenir aux PI administratives de la FP plutôt qu'aux autres catégories de salariés du secteur public. Ce «petit déplacement social» et intergénérationnel, pour reprendre l'expression de Bernard LAHIRE (2006 [2004] : 411-417) est donc un des fondements de ce sentiment d'ascension sociale pour les membres du groupe étudié. On peut d'ailleurs faire l'hypothèse que ces expressions subjectives s'enracinent également dans les trajectoires scolaires montantes entre les générations familiales; à l'image des parcours d'une partie grandissante des jeunes générations issues des fractions supérieures des classes populaires qui accèdent à des diplômes universitaires de premier voire de second cycle (Hugrée, 2009, 2010).

Les données longitudinales du panel d'agents civils de la FPÉ (Insee) complètent cette première vue transversale et rétrospective des parcours des PI administratives de la FP par l'observation des trajectoires professionnelles à l'échelle catégorielle. L'accélération notable, entre les cohortes 1983, 1990 et 1997 des promotions de «catégorie B » en «catégorie A» (respectivement $24 \%, 33 \%$, et $41 \%$ au bout de huit ans d'ancienneté) (Bessière, Pouget, 2007, pp. 58-59) invite alors à penser les dimensions plurielles des sentiments de mobilité ascendante et d'appartenance aux classes moyennes tant semble cohabiter dans une catégorie administrative ou une catégorie socioprofessionnelle identique, des générations marquées par des aspirations, des qualifications et des carrières très différentes. Il a ainsi fallu 20 ans pour que la moitié de la cohorte 1983 de fonctionnaires de «catégorie $\mathrm{B}$ » deviennent «catégorie $\mathrm{A} »$ alors qu'il a fallu moins de 10 ans à celle de 1997 (ibid.). Le «rêve impossible» des fonctionnaires intermédiaires du XIX ${ }^{\mathrm{e}}$ siècle (LE BIHAN, 2008, pp. 223-260) est bien aujourd'hui à portée de main de nombre de leurs homologues contemporains.

Mais les échantillons des enquêtes retenues pour leur étude n'offrent que peu d'occasions de saisir plus précisément les éléments antérieurs à l'entrée dans ces statuts. D'une part, on ne peut pas repérer les éléments concrets qui commandent l'inscription et l'obtention d'un "petit» concours pour ces diplômés de l'enseignement supérieur long. En ce sens, «la carrière et la socialisation concourantes» qui désigne, selon Émilie Biland (2008, p. 499), «les séquences successives, depuis la «décision»de passer le concours, jusqu'aux conséquences de sa réussite (ou de son échec), en passant par le travail (variable) de préparation dans lesquels les candidats sont engagés » restent inaccessibles depuis les enquêtes de la statistique publique. D'autre part, les dimensions les plus scolaires (type de diplôme, options, filières, etc.), liées aux pratiques studieuses et à leur inscription dans le temps, et peut-être davantage aux rapports personnels aux savoirs, aux verdicts scolaires et aux destins professionnels envisagés ne peuvent ici faire l'objet de traitements statistiques du fait de la taille des échantillons ou de l'absence de ces informations.

Il s'agit pourtant de données décisives non seulement pour approcher les causes contemporaines de la réussite ou de l'échec à un concours, mais aussi parce qu'elles sont au fondement d'une estimation subjective, par les individus, de la valeur scolaire et sociale qui précède l'inscription à un concours, sa réussite et l'obtention d'un statut. 
Tableau 1 : Distinguer les PI administratives de la FP des salariés du secteur public en 1993 et en 2003

\begin{tabular}{|c|c|c|c|}
\hline & 1993 (Modèle 1) & 2003 (Modèle 2) & 2003 (Modèle 3) \\
\hline & Coef. & Coef. & Coef. \\
\hline Femme & $0,43 * *$ & 0,14 & 0,17 \\
\hline Homme & Réf. & Réf. & Réf. \\
\hline Moins de 35 ans & 0,17 & $-0,12$ & $-0,08$ \\
\hline Plus de 35 ans & Réf. & Réf. & Réf. \\
\hline Conjoint agriculteur & $-0,27$ & $-0,24$ & $-0,18$ \\
\hline Conjoint artisan, commerçant, chef d'entreprise & $1,23 * * *$ & 0,13 & 0,14 \\
\hline Conjoint cadre & $0,64 * *$ & 0,12 & 0,11 \\
\hline Conjoint profession intermédiaire & $-0,03$ & $-0,07$ & $-0,07$ \\
\hline Conjoint employé & $1,15 * * *$ & 0,18 & 0,18 \\
\hline Conjoint ouvrier & Réf. & Réf. & Réf. \\
\hline Pas de conjoint, Conjoint inconnu & 0,39 & 0,03 & 0,03 \\
\hline Père agriculteur & 0,25 & 0,19 & 0,18 \\
\hline Père artisan, commerçant, chef d'entreprise & $0,62 * *$ & 0,00 & 0,05 \\
\hline Père cadre & 0,24 & $-0,12$ & 0,01 \\
\hline Père profession intermédiaire & $-0,01$ & $-0,02$ & 0,07 \\
\hline Père employé & $0,54 *$ & 0,25 & $0,29 *$ \\
\hline Père ouvrier & Réf. & Réf. & Réf. \\
\hline Père inactif, inconnu & 0,20 & $-0,02$ & 0,14 \\
\hline Bac +2 et plus & $-0,82 * *$ & $0,42 *$ & $0,42 *$ \\
\hline Baccalauréat, brevet professionnel ou équivalent & 0,4 & $1,04 * * *$ & $1,05 * * *$ \\
\hline CAP, BEP & 0,14 & $0,52 * *$ & $0,52 * *$ \\
\hline BEPC seul & $0,77 * * *$ & $1,07 * * *$ & $1,06^{* * *}$ \\
\hline CEP, aucun diplôme, diplôme inconnu & Réf. & Réf. & Réf. \\
\hline Administration nationale & $1,39 * * *$ & $2,28 * * *$ & $2,3 * * *$ \\
\hline Collectivités locales, hôpitaux publics, HLM & $-0,36$ & $0,83 * * *$ & $0,92 * *$ \\
\hline Sécurité sociale, entreprise publique ou nationale, entreprise privé & Réf. & Réf. & Réf. \\
\hline Fonctionnaire & $0,66 * *$ & $-0,17$ & $-0,18$ \\
\hline Contractuel en CDI & $-0,37$ & $-0,1$ & 0,05 \\
\hline Contractuel en $C D D$ ou vacation & Réf. & Réf. & Réf. \\
\hline Premier poste & 0,47 & 0,17 & 0,18 \\
\hline Accès au poste par promotion interne & $0,51 *$ & $0,36 * *$ & $0,38 * *$ \\
\hline Accès au poste par changement de fonction ou de poste (même entreprise) & $0,55 *$ & $0,74 * * *$ & $0,74 * * *$ \\
\hline Accès au poste par changement d'entreprise & Réf. & Réf. & Réf. \\
\hline Cadre 5 ans auparavant & $-3,44 * * *$ & $-2,0 * * *$ & $-2,09 * * *$ \\
\hline Profession intermédiaire 5 ans auparavant & $1,24 * * *$ & $1,44 * * *$ & $1,43 * * *$ \\
\hline Employé 5 ans auparavant & $-1,14 * * *$ & $-0,32 * *$ & $-0,28$ \\
\hline Ouvrier 5 ans auparavant & $-15,31$ & $-1,19 * * *$ & $-1,22 * * *$ \\
\hline Inactif ou inconnu 5 ans auparavant & Réf. & Réf. & Réf. \\
\hline À un ou plusieurs salariés sous ses ordres & $0,77 * * *$ & $0,83 * * *$ & $0,85 * * *$ \\
\hline N'a pas de salariés sous ses ordres & Réf. & Réf. & Réf. \\
\hline Est en contact direct avec le public & & & $-0,37 * * *$ \\
\hline N'est pas en contact direct avec le public & & & Réf. \\
\hline Bien plus élevé que celui de son père & & & 0,29 \\
\hline Plus élevé que celui de son père & & & $0,32 * *$ \\
\hline À peu près le même que celui de son père & & & Réf. \\
\hline Plus bas que celui de son père & & & 0,13 \\
\hline Bien plus bas que celui de son père & & & 0,19 \\
\hline Ne sait pas, NR & & & $-0,55$ \\
\hline Pourcentage de paires concordantes & 89,3 & 83,9 & 84,2 \\
\hline Nombre d'observations & 3105 & 6990 & 6990 \\
\hline
\end{tabular}

Lecture : il s'agit d'un modèle de type logit. On part d'une situation de référence signalée en italique. Le modèle permet d'évaluer, qu'en 1993, être une femme plutôt qu'un homme présentant toutes les caractéristiques de la situation de référence, augmentait toutes choses égales par ailleurs la probabilité d'être PI administrative de la FP plutôt que de ne pas l'être d'un facteur 1,5 $(\exp (0,43)$, soit $57 \%$ de chance de plus $((100 / 1+\exp (-(-5,58+0,43)))$ x100). Dans la seconde colonne, les signes *** indiquent que le paramètre estimé est significatif au seuil de $1 \%$, ** pour un seuil de $5 \%$, * pour un seuil de $10 \%$.

Champ : Salariés actifs du secteur public au moment de l'enquête hors agriculteurs et artisans commerçants chefs d'entreprises cinq années avant l'enquête. Données pondérées.

Sources : enquêtes FQP 1993 et FQP 2003 (Insee) (Diffusion : Centre Maurice Halbwachs). 


\section{"Petits" concours et "grands" diplômés : de nouveaux usages des concours de catégorie $B$ ?}

La question de l'orientation des sortants de l'enseignement supérieur vers les statuts intermédiaires du secteur public est évoquée dans les premières polémiques autour de la valeur des diplômes dans les années 1970 (Lévy-Garboua, 1976; Bourdieu, 1978; Baudelot, Benoliel, Cukrowitz, Establet, 1981, p. 155; BolTANSKI, 1980, JAROUSSE, 1984). Mais, elle prend une acuité nouvelle dans l'université massifiée des années 1990 et 2000, lorsque Stéphane BeAud (2002, p. 11) analyse, d'un point de vue ethnographique, les échecs répétés à l'université et aux concours de deux jeunes (Fehrat et Nassim) d'origine populaire ayant accédé «un peu par effraction» au bac puis au premier cycle universitaire d'études d'Administration économique et sociale. Coincé, à près de trente ans, entre "la peur de s'insérer et la peur de ne pas s'insérer» (Mauger, Vulbeau, 1998), Nassim, qui n'a pas réussi à obtenir son DEUG en 2001, entrevoit alors, de temps à autre, la possibilité de préparer des concours de catégorie $\mathrm{B}$, mais «se pose la question de savoir comment contourner cet obstacle du diplôme plancher pour passer [c] es concours" (2002, p. 295).

Enquêtant, les déterminants socio-économiques d'entrée dans différentes fonctions publiques au début des années 2000 (AUDIER et al., 2004), plusieurs économistes observent que, désormais, «la stratégie individuelle du «sur-diplôme» pour entrer dans la fonction publique apparaît efficace dans les concours étudiés » (Meurs, Audier, 2004, p. 563); de nombreux étudiants ayant anticipé une concurrence accrue de la part des plus diplômés sur certains concours de catégorie A et B. Ces chercheurs identifient ensuite des logiques de candidatures plutôt orientées vers les contenus de la future activité professionnelle pour les plus diplômés, quand les candidats aux niveaux de diplôme plus modestes mettent davantage en avant la sécurité de l'emploi et les conditions de travail. De leur point de vue, les candidats au concours de catégorie B qu'ils étudient se situent à cheval entre ces deux logiques.

La recherche d'Émilie BILAND (2008; 2010a; 2010b) soulève également la question des nouveaux usages des concours, au sein de la FPT. L'auteure souligne ainsi que, dans cette fonction publique, «le concours constitue la voie d'accès au statut de fonctionnaire pour la majorité des cadres, tandis qu'il fonctionne comme une voie de promotion pour une minorité des agents d'exécution»(2010a, p. 60). Elle montre alors que les profils des candidats aux concours de la FPT et de la FPÉ (aux modalités de recrutement historiquement différentes) tendent aujourd'hui à converger pour certains concours. Elle constate que le curriculum des épreuves contemporaines des concours de la FPT s'inscrit désormais plus explicitement dans la continuité de certaines formations universitaires de niveau licence et maîtrise par l'évaluation de compétences scolaires qu'elle qualifie de "générales». Elle note enfin que «la carrière concourante se joue aussi sur la scène familiale» (BILAND, 2008, p. 644-645) tant les concours érigent la disponibilité pour le travail de préparation et de révision en une ressource incontournable de la réussite.

Il s'agit de prolonger ces réflexions en se focalisant sur le cas des concours de B, entrevus depuis une recherche sur les diplômés de l'université issus des milieux populaires et suivis pendant les premières années de leur transition professionnelle (Hugrée, 2009, 2010). L'enquête ethnographique souligne alors l'existence de trajectoires contrariées, et non seulement interrompues, d'accès à l'emploi public qualifié. Chez les candidats ayant échoué aux concours de catégorie A de la fonction publique, de telles transitions se présentent dans les premiers temps en «demi-teinte». Mais c'est pourtant dans ces moments biographiques un peu troubles, et parfois longs de plusieurs années, que les ressources scolaires, familiales et symboliques leur permettent d'accéder, par le biais d'un concours moins sélectif, à des débuts de carrière sensiblement différents de ceux enquêtés par Stéphane Beaud. Le seuil que constituent la licence et les anciens diplômes de deuxième cycle universitaire, se révèle alors au fondement de l'effet de cliquet qui fait se séparer, après plusieurs années, les différents destins des enfants de la seconde explosion universitaire.

L'étude détaillée de la trajectoire scolaire, universitaire et professionnelle de Camille, issue des fractions ascensionnelles des milieux populaires, titulaire d'une licence d'histoire-géographie et reçue au concours de conseiller ANPE(9) en 2006, permet de compléter la connaissance quantitative des déterminants de cette rencontre, désormais probable, entre des diplômés de l'enseignement supérieur long et général et des concours de catégorie $\mathrm{B}$. Et, derrière la singularité du parcours de cette enquêtée et de ses rapports à l'université, au concours et à l'enquête, c'est finalement l'articulation entre les cheminements biographiques et la nouvelle configuration scolaire de l'emploi public qui se donne à voir. Ce portrait permet en effet d'envisager le parcours d'insertion d'une diplômée de second cycle

(9) Avant la fusion avec les Assedics et la création de Pôle Emploi, les conseillers ANPE étaient des fonctionnaires recrutés par voie de concours B. Ils appartiennent au groupe des PI administratives de la FP dans la nomenclature PCS de 2003, et sont classés dans la profession 451e «Autres personnels administratifs de catégorie B de l'État (hors Enseignement, Patrimoine, Impôts, Trésor, Douanes)», http://www.insee.fr/fr/ methodes/default.asp?page $=$ nomenclatures/pcs2003/n4_451e. htm 
universitaire, non seulement au regard de l'état du «marché» des concours, mais aussi de ses pratiques scolaires et universitaires (socialisation à la discipline, intériorisation des exigences des concours). On découvre alors que cette carrière concourante contrariée se comprend par des reconversions successives des aspirations sociales et un parcours scolaire «honorable» (HugréE, 2009, 2010) qui rendent acceptables et même finalement enviables l'accès à un statut intermédiaire moins prestigieux que ceux premièrement envisagés.

\section{Une socialisation scolaire et universitaire inachevée}

Camille est la fille aînée d'un ouvrier qualifié («traceur») qui a commencé en apprentissage aux chantiers de l'atlantique de Saint-Nazaire. M. Houdon a connu, aux dires de sa fille, une progression à la fin de sa carrière, puisqu'il travaillait "dans un petit bureau avec un ordinateur» et n'était plus, selon l'expression de Camille, «à quatre pattes sur les tôles. » La mère de Camille est, quant à elle, aide éducatrice dans un Institut médico éducatif de la ville et syndiquée à la CGT. Le premier entretien s'est avéré un des plus compliqués de tous ceux réalisés avec elle entre 2005 et 2009. D'abord nerveuse, riant dès qu'elle évoque la moindre anecdote, Camille passe en fait sous silence la plupart des éléments gênants de sa biographie. Il faudra par exemple revenir à plusieurs reprises sur son vécu des échecs successifs au concours du Certificat d'aptitude au professorat de l'enseignement du second degré (CAPES), pour finalement comprendre que ses réponses furtives sont déjà lourdes de sens. En fait, ce premier entretien la «cueille» dans un moment délicat de sa biographie. Passant pour la quatrième fois son concours de recrutement des professeurs des écoles (CRPE), Camille a, à l'époque, 29 ans et supporte difficilement la comparaison de statut avec ses camarades. Cette année est même pour elle une année décisive, puisqu'elle avoue qu'après sept ans de préparation de concours, elle n'envisage pas échouer une fois de plus. L'entretien lui rappelle donc l'enjeu de cette année où l'horizon d'un poste tant espéré lui semble plus proche que jamais et en même temps encore bien incertain.

Nos nombreuses rencontres et entretiens ont par la suite permis un retour sur certains éléments de son dossier scolaire et la lecture commentée de ses bulletins; archives personnelles (conservées par sa mère) qui permettent ici de préciser certains aspects de ces scolarités honorables, c'est-à-dire distantes des canons de l'excellence scolaire mais aussi éloignées des plus classiques échecs scolaires en milieux populaires.

Les résultats de Camille, au sortir de l'école primaire, présentent un niveau «satisfaisant» ou «très satisfaisant» en «français» et «calcul». Son institutrice évalue, en ces termes, son parcours en CM2 : «Bon travail. Élève timide, mais sérieuse. Devrait réussir en sixième»; là où elle indique un niveau de classe «assez moyen, à quelques exceptions près. » Bonne élève lors de l'entrée au collège public de son quartier, Camille (comme sa sœur cadette), est inscrite, sur les recommandations de sa mère, en classe d'Allemand première langue en sixième puis en latin en quatrième. Les appréciations et les notes de troisième révèlent un «travail sérieux», "des résultats satisfaisants». Ses résultats en mathématiques et en sciences physiques semblent confirmer ses grandes difficultés dans ces matières, ce que ne manquent pas de souligner l'ensemble du conseil de classe et son enseignant de mathématiques : "Résultats faibles. Il ne faut pas abandonner les mathématiques en troisième.» À son entrée au lycée, Camille se situe dans la moyenne de sa classe de seconde et son enseignant principal souligne «des faiblesses passagères mais un niveau d'ensemble convenable.» L'orientation de Camille dans l'ancêtre de la filière littéraire est marquée par un goût scolaire pour les langues, l'histoire-géographie, un net rejet des mathématiques et par le désir précoce d'être enseignante en primaire. Quelques jours avant les épreuves du baccalauréat, son conseil de terminale énonce un "avis assez favorable» à son sujet, ce qui la place, selon cette échelle, dans la première moitié des candidats au baccalauréat A de son établissement. Lors de l'entretien, Camille explique qu'elle avait fait le pari de la mention, en raison de ses résultats en histoiregéographie et des incitations de son enseignante de l'époque à « $y$ croire». Elle hésite au moment de l'entretien à qualifier ce soutien : «à cause d'elle... Enfin grâce à elle plutôt $»$. Cette hésitation dans l'appréciation rétrospective de sa relation avec une enseignante importante dans son parcours résonne doublement. D'une part, elle renvoie à son rattrapage au baccalauréat quand cette enseignante l'incitait à croire possible une mention. D'autre part, elle évoque les regrets de Camille à s'être lancée, un peu sur les conseils de cette enseignante, dans la préparation du CAPES d'histoire-géographie et à avoir cru qu'une issue favorable était également possible. Malgré tout, fidèle à sa «vocation» et à son goût pour la matière où elle a connu ses meilleurs résultats au lycée, Camille s'inscrit à la faculté d'histoire à Nantes. Elle redouble alors sa première année, faute d'une mobilisation suffisante: «Je n'allais pas beaucoup en cours. Et, je faisais juste ce qu'il fallait pour avoir des contrôles continus potables, et pour avoir une super-note au partiel...» Se ressaisissant lors de sa deuxième première année, elle est acceptée en deuxième année, après avoir dû repasser deux modules au rattrapage. Elle décroche finalement son DEUG d'histoire à la première session mais sans mention. Elle s'inscrit alors en licence et choisit des enseignements optionnels réputés difficiles, tels que «sciences auxiliaires du médiéval» ou «histoire des religions». En dépit des réputations de ces deux 
options, («ça faisait halluciner tout le monde que je fasse ça»), Camille ne les envisage pourtant pas dans la perspective de continuer en maitrise ou de préparer le CAPES mais au contraire sur le mode d'une curiosité intellectuelle et personnelle. C'est aussi dans cet enseignement qu'elle se découvre distante d'une des dimensions du métier d'historien : "Le fait de faire de l'histoire des religions, je me suis rendu compte que le travail d'archive, ça me gavait!»; ce qui conforte son aspiration à l'enseignement. L'apparent désintérêt qui préside aux choix d'options de Camille semble en fait révélateur d'un rapport plus général à ses études supérieures et à sa discipline universitaire, entrevues non pas comme un moyen de se préparer efficacement au CAPES d'histoire-géographie (elle n'a d'ailleurs pas choisi les options de géographie) mais comme une forme d'émancipation intellectuelle et culturelle, distante des dimensions les plus stratégiques et formelles de la préparation d'un concours.

\section{Une carrière concourante longue et contra- riée}

Lors de sa première année d'IUFM, Camille ne semble pas complètement mobilisée en vue du concours : "je savais que ça n'allait pas être facile, mais je ne me suis pas donnée à fond non plus. La majorité des gens m'avaient dit que le CAPES, tu l'as rarement du premier coup. » Non admissible à sa première tentative, en 1999, Camille tente à nouveau de préparer le concours à l'IUFM, en vain. «J'étais classée superbas! Je me suis ramassée à l'entretien professionnel, j'ai eu 0,5! [rire]. Apparemment c'est courant. Je suis tombée sur «l'utilisation des concepts en histoire». Et, en fait, si j'avais lu les "Dix leçons sur l'histoire» [sic] d'Antoine Prost, j'aurais pu m'en sortir. Mais pas de bol, je ne l'avais pas lu! [rires] Donc, c'est dommage."

Pour comprendre ces échecs répétés au concours, en dépit d'une mobilisation certaine dans le travail de révision et d'une présence continue aux cours, il faut tout d'abord évoquer la baisse continue du nombre de postes mis au concours externe du CAPES d'histoire-géographie (le taux de sélection entre les candidats présents évolue autour de $10 \%$ lors de ses années de candidatures). Mais, dans ce moment de concurrence universitaire accrue, Camille est aussi confrontée aux limites de sa pratique étudiante passée. Indice de sa distance aux réquisits de l'exercice et du caractère inachevé de sa socialisation disciplinaire (MILLET, 2003) à l'histoire-géographie, Camille indique ne pas avoir lu le classique des révisions de concours en histoire-géographie qu'est Douze leçons sur l'histoire et écorche, au passage, le titre de l'ouvrage. Avec l'aide d'une amie dans la même situation, elle décide alors de se rabattre sur le concours de recrutement des professeurs des écoles avec une ambition non dissimulée. Elle devient également, à partir de 2002, surveillante d'externat dans un collège de Nantes pour financer sa préparation au concours. Après une première tentative manquée au CRPE, Camille s'inscrit au Centre national des études à distance (CNED). Elle tente alors depuis 2005 de passer ce concours dans l'académie de Versailles, connue des étudiants pour être moins sélective. Mais, là encore, sa candidature échoue. À l'époque, la baisse progressive de ses aspirations trouve son salut dans la nouvelle relation amoureuse qu'elle a avec Icham, un ingénieur informaticien en poste depuis trois ans sur la région parisienne. La joie d'être admissible dans l'académie de Versailles en 2005 est de courte durée puisqu'elle ne figure «même pas» sur la liste complémentaire des postes mis au concours; liste qui, il y a encore peu de temps, permettait à une proportion parfois importante de recalés au concours d'accéder en cours d'année à un poste d'enseignant, sous le statut de stagiaire de la fonction publique.

Refusée une dernière fois au CRPE en 2005, Camille arrive aussi au terme de son contrat de surveillante d'externat dans son collège. Ne se voyant alors proposer qu'un mi-temps sur le nouveau statut d'assistante d'éducation, elle s'inscrit comme demandeuse d'emploi, à l'Agence nationale pour l'emploi (ANPE) et se présente alors dans toute une série de concours de catégorie $\mathrm{B}$ : celui de conseiller ANPE, celui de contrôleur des impôts, de contrôleur des douanes, et enfin celui de contrôleur du travail. Elle se présente aux épreuves du concours de conseiller ANPE de l'Île-de-France où réside Icham, son compagnon rencontré un an auparavant, et où le nombre de places ouvertes (300) lui fait apparaître ce concours régional comme accessible : «Donc, voilà. Je n'ai rien préparé du tout. Et j'y suis allée comme ça, les mains dans les poches.» Camille est alors surprise du nombre de candidats présents aux épreuves : «Et quand j'ai vu le monde, je me suis dit : "Oh là!». Il y a plus de monde que pour prof des écoles [...]» Elle est finalement retenue pour les épreuves orales en janvier 2006. Celles-ci se séparent entre «une analyse de situation» d'un quart d'heure et un entretien pré-professionnel de trois quarts d'heure avec le jury. Sa conseillère ANPE de l'époque lui explique le mode opératoire d'un concours dont les exercices ne semblent pas connus de tous les candidats. Camille est finalement appelée au mois de mars 2006 pour un poste dans une agence ANPE de Garges-lès-Gonesse, d'où son compagnon est originaire. On mesure à travers les propos de Camille et ses résultats aux différentes épreuves que ce sont celles reposant sur des dispositions culturelles et scolaires transversales (argumentation, mise en ordre de sa trajectoire biographique) et non sur des savoirs spécifiques, qui ont contribué à son succès à ce concours. Décrochant finalement un poste, elle accueille cette nouvelle avec un soulagement total qui résonne comme une véritable réhabilitation d'elle-même. Ce point est notamment visible dans le fait qu'elle répète, à plusieurs reprises dans l'entretien, avoir réussi un concours 
qu'elle n'a «vraiment pas travaillé du tout». Mais, plus de six mois après son recrutement, c'est aussi avec l'espoir de pouvoir, "grimper à l'interne», qu'elle envisage sa carrière dans l'ANPE.

On comprend que la relation entre les diplômes de Camille et son emploi de conseillère ANPE, plusieurs années après la sortie de l'université, est envisagée à travers une pluralité d'éléments qui ne se réduisent pas à l'entrée dans un statut en perte de prestige, peu à peu distancié des emplois qualifiés de la FPÉ. Comme elle le souligne dans l'entretien, le statut et les revenus de son compagnon, tout comme sa longue quête d'un emploi de fonctionnaire de catégorie A, fonctionnent comme de véritables modérateurs dans l'appréciation de son désajustement salarial.

Ce suivi ethnographique fait comprendre comment s'est petit à petit produit l'ajustement entre une aspiration à la mobilité professionnelle et sociale et une position de fonctionnaire intermédiaire, puisque Camille occupe, aujourd'hui encore, ce premier poste. Un peu plus d'un an après nos entretiens chez ses parents, elle est désormais mère d'une petite fille née en septembre 2007. Du point de vue professionnel, elle se définit à présent comme une conseillère ANPE «cordiale et humaine» avec les demandeurs d'emploi, ce à quoi elle ajoute : «mais, il ne faut pas me prendre pour une bille!», indiquant par là, qu'elle est attachée à une forme de bonne volonté de la part des chômeurs qu'elle suit dans leur recherche d'emploi. Si elle déclare apprécier son rôle de conseillère et les activités qui y sont liées, elle dit également détester tenir le poste d'accueil qui «trie» les demandes des demandeurs d'emplois : "C'est chiant. T'es en première ligne, t'es le bureau des pleurs!» Indice d'une socialisation professionnelle en cours, Camille s'est d'ailleurs syndiquée et a fait grève en 2007 contre le projet de fusion des Assedics et de l'ANPE : «Nous, on n'est pas flics, nous, on ne contrôle pas» dit-elle alors en entretien. Un nouvel entretien en 2009, m'apprend que Camille et sa famille ont quitté leur premier appartement mais résident toujours à Garges-lès-Gonesse car ils bénéficient désormais d'un logement neuf, réservé aux employés de l'ANPE, face aux grands ensembles des années soixante. Contente de son nouveau logement, elle indique pourtant avoir abandonné l'idée d'habiter «ici», expression qui désigne à la fois sa commune de résidence et les grands ensembles défraichis visibles depuis son salon. Camille guette en fait les postes accessibles dans la ville du Mans qui permettraient à Icham de garder son emploi à Paris.

Lors d'un ultime entretien, nous évoquons également ce qu'est devenue son aspiration initiale à «grimper à l'interne». Elle revient, avec scepticisme, sur les effets de la fusion Assedics/ANPE sur la définition des postes qu'elle envisageait pour la suite de sa carrière.
Elle détaille alors plus en avant les reconfigurations qu'ont occasionnées les différentes phases de la fusion Assedic/ANPE et leurs effets sur l'organisation de son agence et de son poste. Et, elle qui a tant lutté pour obtenir le statut de fonctionnaire, finit aujourd'hui par peser le pour et le contre avec le secteur privé : «En fait, dans le privé, je serais peut-être mieux payée, donc... Là, notre boulot change et en termes de rémunération... C'est quand même pas terrible.» Et, interrogée sur d'éventuels regrets sur ses années universitaires et sa quête des concours, Camille répond: " Je ne regrette pas $d u$ tout d'avoir fait des études d'histoire. Par contre, j'aurais peut-être dî renoncer avant... [rires] au concours! Autant, le concours ANPE, je ne regrette pas du tout. C'est vraiment un boulot qui me plaît. Mais prof des écoles, et en plus en le passant ici [à Garges-lès-Gonesse] sur la fin... Quand je vois déjà les gosses des voisins... Je me dis non. Je n'aurais pas pu. [...] C'est vrai que là, quand je sors du travail, c'est fini. Quand je vais en vacances, je n'ai pas à me dire: "est-ce que j'ai pensé à mon "cahier-journal» et des trucs comme ça». [...] Moi, les vacances, c'est les vacances.»

$$
\text { * * }
$$$$
*
$$

Les fonctionnaires intermédiaires qui émergent de cette double analyse, statistique et ethnographique, semblent à la fois traversés par une érosion de leur prestige et une élévation du niveau des diplômes des impétrants. Les variations des échelles et des focales d'observations permettent alors d'interroger les dimensions multiples de la configuration qui régit aujourd'hui l'accès à des emplois publics intermédiaires sans céder au seul schème de l'inflation scolaire (PASSERON, 1982; DURU-BELLAT, 2006) dont les limites sont de plus en plus soulignées (MAURIN, 2007; Coulangeon, 2011; Poullaouec, 2011).

D'un côté, le constat d'une polarisation interne rend compte des multiples recompositions qui font s'éloigner haut, milieu et bas du salariat public. De l'autre, l'analyse de la trajectoire d'accès à ce type d'emploi d'une diplômée de second cycle universitaire objective les conditions scolaires et sociopolitiques qui font désormais se rapprocher des étudiants à la socialisation scolaire inachevée des emplois administratifs de catégorie B. Tout se passe comme si, finalement, les carrières concourantes contrariées de ces étudiants régulaient, de la même façon que les carrières longues de promotion des emplois publics d'hier, les équilibres qui organisent ces professions; contribuant par là à maintenir la position objective de ces professions entre le «petit» salariat public et son encadrement.

Mais, on le voit à travers le portrait de Camille, les transformations organisationnelles et statutaires qui 
traversent aujourd'hui les emplois publics ne sont pas sans conséquences sur l'identité sociale et au travail des fonctionnaires étudiés. Elles contribuent, avec les transformations démographiques évoquées plus haut, à la déstabilisation de la respectabilité des membres de ce groupe, construit comme un idéaltype historique des classes moyennes françaises (Chauvel, 2006) consubstantiel du développement de l'État social (Bourdieu, 1979) et désigné comme une clientèle politique traditionnelle des partis de gauche (Rouban, 2009, p. 74). 


\section{Bibliographie}

Amossé T. (2011), «Cadres/non cadres : une frontière toujours consistante» in Bouffartigue P., Gadea C., Pochic S. (dir.), Cadres, classes moyennes : vers l'éclatement? Paris, Armand Colin, pp. 32-45.

Audier F. (responsable scientifique), Di Paola V., Lambert M., Meurs D., Moullet S., Perez C. (2004), «Les déterminants des entrées dans les fonctions publiques : parcours du combattant ou pis-aller?», Convention MENRT 2002010, MATISSE/Université Paris 1, ERMES/Université Paris 2, LEST/CNRS, CEREQ, Avril.

Baudelot C., Benoliel R., Cukrowitz H., Establet R. (1981), Les étudiants, l'emploi, la crise, Paris, Maspéro.

Beaud S. (2002), $80 \%$ au bac... et après. Les enfants de la démocratisation scolaire, Paris, La Découverte.

Beaud S. (1996), «L'usage de l'entretien en sciences sociales. Plaidoyer pour l'entretien ethnographique», Politix, no 35, septembre, pp. 233-234

Bessière S., Pouget J. (2007), «Les carrières dans la fonction publique d'État. Premiers éléments de caractérisation », in Les salaires en France, Insee, pp. 51-66.

Beż̀s P. (2009), Réinventer l'État. Les réformes de l'administration française (1962-2008), Paris, PUF.

BILAND É. (2008), Concours territoriaux et institutionnalisation de l'emploi public local (Années 1970 - Années 2000), Thèse de doctorat de Sociologie, EHESS.

Biland É. (2010a), «Carrières concourantes. Éléments sur la socialisation par les concours administratifs territoriaux», Terrains et travaux, $\mathrm{n}^{\circ}$ 17, pp. 57-73.

BiLAND É. (2010b), «Les ambiguïtés de la sélection par concours dans la fonction publique territoriale : une institutionnalisation inachevée», Sociologie du travail, no 52, pp. 172-194.

BolTANSKi L. (1980), «Les universités, les entreprises et la multiplication des salariés bourgeois, 1960 - 1975 », Actes de la recherche en sciences sociales, $\mathrm{n}^{\circ} 34$, pp. 17-44.

BounAKHLA N. (2009), «Les recrutements externes dans la fonction publique de l'État en 2007», in Direction générale de l'Administration et de la Fonction Publique, Rapport Annuel de la fonction publique. Faits et chiffres 2008-2009, Paris, La Documentation française.

Bourdieu P. (1978), «Classement, déclassement, reclassement», Actes de la recherche en sciences sociales, $\mathrm{n}^{\circ} 24$, pp. 2-22.

Bourdieu P. (1979), La distinction. Critique sociale du jugement, Paris, Éditions de Minuit.

Coulangeon P. (2011), «Les sociétés malades de leur école?», La Vie des idées, 8 mars, URL : http://www. laviedesidees.fr/Les-societes-malades-de-leur-ecole.html
Chenu A. (2005 [1994]), Sociologie des employés, Paris, La Découverte.

Deauvieau J., Dumoulin C. (2009), «Qui sont les «professions intermédiaires»? Morphologie, rapport au travail et sentiment d'appartenance de classe», in Guérin-Pace F., Samuel O., Ville I. (coord.), La diversité des appartenances. L'enquête Histoire de vie sur la construction des identités, Ined.

Deauvieau J., Dumoulin C. (2010), «La mobilité socioprofessionnelle des professions intermédiaires, fluidité, promotions, déclassement», Économie et statistique, $\mathrm{n}^{\circ} 431$ - 432, pp. 57-72.

Di Paola V., Moullet S. (2003), «L'emploi public et les trajectoires d'insertion des jeunes», Économie et Statistique, no 369-370, pp. 49-74.

Duru-Bellat M. (2006), L'inflation scolaire. Les désillusions de la méritocratie, Paris, Seuil.

Duru-Bellat M., KiefFer A. (2006), «Les deux faces - objective/subjective - de la mobilité sociale», Sociologie du travail, no 48, pp. 455-473.

GollaC S. (2005), «La fonction publique : une voie de promotion sociale pour les enfants des classes populaires?», Sociétés contemporaines, no 58, pp. 41-63.

Goux D. (2010), «L'enquête Formation Qualification Professionnelle : une source inépuisable pour la recherche en sciences sociales », Économie et statistique, no 431-432, pp. 3-11.

HugréE C. (2009), «Les classes populaires et l'université : la licence... et après?», Revue Française de Pédagogie, Mai-Juin, no 167, pp. 47-58.

Hugrée C. (2010), ««Le Capes ou rien?» Parcours scolaires, aspirations socialesetinsertions professionnelles du «haut» des enfants de la démocratisation scolaire», Actes de la recherche en sciences sociales, $\mathrm{n}^{\circ} 183$, Juin, pp. 72-85.

JAROUSSE J.-P. (1984), «Les contradictions de l'université de masse dix ans après (1973-1983)», Revue française de sociologie, Vol. 25, pp. 191-210.

JoIN-LAMBERT O. (2005), «Quand l’État ajuste. Receveurs et receveuses des Postes sous la IVe République», Le mouvement social, no 212, juillet-septembre, pp. 11-33.

LAhire B. (2006 [2004]), La culture des individus. Dissonance culturelle et distinction de soi, Paris, La Découverte.

LÉVy-Garboua L. (1976), «Les demandes de l'étudiant ou les contradictions de l'université de masse», Revue Française de Sociologie, no XVII, pp. 53-80.

Le BiHAn J. (2008), Au service de l'État. Les fonctionnaires intermédiaires au XIXe siècle, Rennes, PUR.

MAURIN É. (2007), La nouvelle question scolaire. Les bénéfices de la démocratisation, Paris, Seuil. 
Meurs D., Audier F. (2004), «Qui se présente dans la fonction publique et pourquoi? Premiers résultats d'enquêtes spécifiques sur les candidats à différents concours de la fonction publique d'État », Revue française d'administration publique, no 111, pp. 547-566.

Millet M. (2003), Les étudiants et le travail universitaire. Étude sociologique, Lyon, PUL.

Monso O., Thévenot L. (2010), «Les questionnements sur la société française pendant quarante ans d'enquêtes Formation et Qualification, Professionnelle», Économie et Statistique, no 431-432, pp. 13-36.

Passeron J.-C. (1982), «L'inflation des diplômes. Remarques sur l'usage de quelques concepts analogiques en sociologie», Revue française de sociologie, $\mathrm{n}^{\mathrm{o}} 23$, pp. 551-584.

Passeron J.-C., Revel J. (2005) (dir.), Penser par cas, Paris, Éditions de l'École des hautes études en sciences sociales.
Pelage A., Poullaouec T. (2009), «La France «d'en bas» qu'on regarde «d'en haut»», in Guérin-Pace F., Samuel O., Ville I. (coord.), En quête d'appartenances. L'enquête Histoire de vie sur la construction des identités, Ined, Paris.

Poullaouec T. (2011), «L'inflation des diplômes. Critique d'une métaphore monétaire» in Millet M., Moreau G., La société des diplômes, pp. 37-49, Paris, La Dispute.

RAYNAUd P. (2003), «L'emploi publique est tiré par la fonction publique territoriale», Économie et statistique, no 369-370, pp. 75-92.

Rouban L. (2009), La fonction publique, Paris, Repères.

Singly (de) F., Thélot C. (1989), Gens du public, gens du privé. La grande différence, Paris, Nathan.

\section{Sources statistiques}

Formation, qualification professionnelle (FQP) - 1993 - (1993) [fichier électronique], INSEE [producteur], Centre Maurice Halbwachs (CMH) [diffuseur].
Formation, qualification professionnelle (FQP) - 2003 - (2003) [fichier électronique], INSEE [producteur], Centre Maurice Halbwachs (CMH) [diffuseur]. 